\title{
Desplazamiento forzado como resultado del cambio climático: Un desafío para el derecho internacional de los refugiados
}

\author{
Forced displacement as a result of Climate Change: \\ New challenges for International Refugee Law
}

\section{Beatriz Romero Cruzat (iD) \\ Investigadora independiente, Chile}

\author{
Francisco Bustos Bustos (iD \\ Universidad de Chile, Chile
}

RESUMEN El derecho internacional de los refugiados, nacido producto de la experiencia histórica de la Segunda Guerra Mundial en la forma de la Convención sobre el Estatuto de los Refugiados (1951), busca proteger a las personas de la persecución por motivos políticos, raciales, nacionales, culturales, religiosos, de género u otros reconocidos universalmente como inaceptables, sea a nivel de persecución individual en los términos de la Convención de 1951, o grupal, en los términos dispuestos por la Declaración de Cartagena (1984). Esta noción de protección internacional derivada del actuar directo del ser humano se ve problematizada a propósito del grupo de personas, cada vez mayor, que se desplaza a raíz del cambio climático, hipótesis que el derecho internacional de los refugiados parece no comprender, y que queda fuera de su marco normativo, aun cuando la vulneración de derechos a la que se ven enfrentadas estas personas resulte del todo evidente. El propósito de este trabajo es reconstruir el marco normativo que podría regular estas nuevas necesidades de protección a la luz del derecho internacional de los derechos humanos, con miras a responder la pregunta por la posibilidad hacer aplicable la protección en el derecho chileno. Así, revisaremos los instrumentos internacionales y el derecho consuetudinario existente, y distintas formas jurídicas para hacer frente a la problemática de las personas que buscan protección por motivo de las consecuencias del cambio climático, a través de la protección complementaria, el principio de no devolución y la prohibición de expulsiones colectivas. Argumentamos que - aun cuando Chile debería legislar al respecto- existen en nuestro ordenamiento instrumentos ya ratificados, que configuran un marco regulatorio de protección aplicable en el marco del derecho internacional de los derechos humanos y de los refugiados. 
PALABRAS CLAVE Derecho internacional de los refugiados, desplazamiento forzado, cambio climático, derecho internacional de los derechos humanos.

ABSTRACT International refugee law, born as a result of the historical experience of the Second World War, in the form of the Convention Relating to the Status of Refugees (1951), seeks to protect people against persecution for political, racial, national, cultural, religious, gender, or others universally reasons recognized as unacceptable, whether at the level of individual persecution under the terms of the 1951 Convention, or group, under the terms provided by the Cartagena Declaration (1984). However, this notion of international protection derived from the direct action of the human being is problematized with regard to the growing group of people who are displaced as a result of climate change, a hypothesis that international refugee law does not seem to include, remaining outside its regulatory framework, even when the violation of rights that these people are facing is completely evident. Considering this, the purpose of this work will be to reconstruct the normative framework that could regulate these new protection needs in the light of international human rights law, with a view to analyzing the feasibility of applying Chilean regulation to these hypotheses. Thus, we will review international instruments and customary law available, to evaluate different legal forms that could protect those who have been displaced as a result of climate change, through complementary protection, the principle of non-refoulement and the prohibition of collective expulsions; arguing that - even when there is no specific legislation in this regard- we already have ratified instruments in our legal system that could serve these purposes.

KEYWORDS International Refugee Law, forced displacement, climate change, International Human Rights Law.

\section{Introducción}

El clima cambiante, las inundaciones y las sequías amenazan cada vez más la seguridad y los medios de vida de las personas en muchos lugares del planeta. Esto está llevando a muchas familias a considerar si pueden quedarse donde están o tratar de vivir en otro lugar. -Koko Warner, Secretaria del Área de Migración de la Convención de Naciones Unidas sobre Cambio Climático

En el marco de lo que hubiera sido la Conferencia de Naciones Unidas sobre el Cambio Climático de 2019 ( $\mathrm{COP}_{25}$ ) en Chile, y considerando que el cambio climático nos afecta a todos en múltiples formas, parece relevante analizar la situación jurídica de aquellas personas que se verán obligadas a desplazarse de sus hogares, sea porque los mismos dejan de existir - por el aumento del nivel del mar u otra situación análoga-, o bien, porque esos hogares dejen de ser aptos para la vida humana. 
El mundo está siendo testigo del mayor número de desplazamientos del que se haya tenido registro. Según cifras del Alto Comisionado de las Naciones Unidas para los Refugiados (ACNUR), 70 millones de personas ${ }^{1}$ se han visto obligadas a abandonar sus hogares por una serie razones. Los conflictos políticos e ideológicos es la principal razón, pero no es la única.

El desplazamiento forzoso como consecuencia de desastres naturales es un fenómeno que toma cada vez más fuerza. «Un promedio anual de 21,5 millones de personas han sido forzosamente desplazadas cada año, desde 2008, por amenazas relacionadas con el clima, como inundaciones, tormentas, incendios forestales, temperaturas extremas. Miles de personas huyen de sus hogares en el contexto de amenazas de evolución lenta, como las sequías y la erosión costera relacionadas con aumentos del nivel del mar». ${ }^{2}$ A pesar de lo contundente de esta cifra, el marco legal internacional no contempla, al menos explícitamente, hipótesis de protección frente a desplazamientos motivados por hechos de esta naturaleza. En este sentido, se emplea la denominación "personas desplazadas en el contexto de desastres y cambio climático».3

En las líneas siguientes estudiaremos, a la luz de los instrumentos del derecho internacional de los derechos humanos, las posibilidades de otorgar protección a quienes se ven obligados a migrar de su Estado o lugar de residencia habitual por razones asociadas a desastres naturales, para efectos de esbozar una respuesta a esta pregunta compleja en el ámbito del ordenamiento jurídico chileno.

Este ensayo se desarrollará en tres secciones. En la primera, revisaremos el marco regulatorio internacional de la protección internacional. El desarrollo de esta sección comprende el concepto de protección internacional y cómo es que el estatuto de refugiados y la protección complementaria forman parte de ella. En la segunda sección analizaremos el concepto de cambio climático y la afectación de derechos humanos que puede generar; el desplazamiento forzado es una de ellas. Finalmente, en la tercera y última sección abordaremos el cambio climático como hipótesis de protección internacional empleando instrumentos generales de protección. El análisis comenzará con la revisión del marco jurídico aplicable, para continuar con el análisis de algunas de las fuentes jurídicas que podrían servir de base para justificar el otorgamiento de protección internacional en estos casos, dado el contexto político que tiene lugar en la actualidad.

1. ACNUR, «El desplazamiento global supera los 70 millones de personas y el Alto Comisionado de la ONU para los Refugiados pide más solidaridad», 19 de junio de 2019, disponible en http://bit. ly/3rgBgW7.

2. ACNUR, «Preguntas frecuentes sobre el desplazamiento causado por el cambio climático y los desastres naturales», 6 de noviembre de 2016, disponible en http://bit.ly/3mEPDA7.

3. ACNUR, «El cambio climático y los desastres provocan cada vez más desplazamientos», 28 de junio de 2019, disponible en http://bit.ly/3pcwile. 


\section{Marco regulatorio de la protección internacional}

\section{Definición de protección internacional y el principio de no devolución}

Todo Estado tiene la responsabilidad de proteger a sus ciudadanos y a todos quienes residan o se encuentren en su territorio, velando porque sus derechos fundamentales sean resguardados y respetados en el orden interno. Sin embargo, esto no siempre ocurre y por diversas situaciones los Estados incumplen tal deber. En ese momento, surge la necesidad de protección internacional, la que ha sido definida por el ACNUR (2005a) como «todas las actividades destinadas a asegurar el acceso igualitario y el disfrute de los derechos a mujeres, hombres, niñas y niños bajo la competencia del ACNUR, de acuerdo con los instrumentos legales pertinentes, incluyendo el derecho internacional humanitario, los derechos humanos y el derecho internacional de los refugiados».

Lo anterior, supone que la protección internacional deberá otorgarse a una persona cuando sus derechos humanos se vean amenazados o vulnerados en su país de nacionalidad o residencia habitual y, al mismo tiempo, cuando la protección de ese Estado sea considerada insuficiente o inexistente, de modo tal que la protección de un tercer Estado se torne necesaria (Reyes Canales y Romero, 2018: 6).

Las causas de dichas vulneraciones pueden deberse a múltiples circunstancias, según ha indicado Comité Ejecutivo del ACNUR (2005b: 1): «generalmente se relacionan con la persecución, las amenazas a la vida y la seguridad personal, el conflicto armado, los disturbios públicos significativos u otras catástrofes ocasionadas por el ser humano. Los desastres naturales o ecológicos o la inseguridad provocada por la apatridia son causas adicionales de esta situación». En definitiva, la protección internacional comprende dos elementos: en primer lugar, el reconocimiento que la persona se encuentra en necesidad de protección, y, en segundo término, los derechos que se adjuntan tras dicho reconocimiento.

Cabe señalar, que la protección internacional de una persona comienza al asegurar su admisión en el país de asilo, continúa con la concesión de protección y el consiguiente respeto de sus derechos humanos fundamentales, incluyendo el derecho a no ser regresado de manera forzada a un país donde su seguridad o su supervivencia se encuentren amenazadas y termina solo con la obtención de una solución duradera (ACNUR, 2005a: 18).

La protección internacional descansa sobre el principio de no devolución o principio de non-refoulement. ${ }^{4}$ Este constituye el principal fundamento del régimen in-

4. El término tiene su origen en la voz francesa refouler (hacer retroceder). Sobre el mismo, véase ACNUR, «Opinión consultiva sobre la aplicación extraterritorial de las obligaciones de no devolución en virtud de la Convención sobre el Estatuto de los Refugiados de 1951 y su Protocolo de 1967», de 26 de enero de 2007, disponible en https://bit.ly/38PACGH; Comité contra la Tortura, «Observación General 
ternacional de protección de los refugiados. En virtud de este principio, se prohíbe el retorno forzado de las personas que puedan verse expuestas al riesgo de sufrir vulneraciones graves a sus derechos fundamentales.

El primer instrumento que hizo referencia a este principio fue la Convención sobre el Estatuto Internacional de los Refugiados de $1933,{ }^{5}$ mientras que la Convención de 1951 fue el primer tratado internacional en codificarlo. Con el tiempo, otros cuerpos normativos lo han incorporado: es el caso de la Convención Americana sobre Derechos Humanos de 1969, la Convención de la Organización de la Unión Africana de 1969 que regula aspectos específicos sobre refugiados, y la Declaración de Cartagena de 1984 .

Este principio se encuentra consagrado en el artículo 33.1 de la Convención de 1951, en los siguientes términos: «Ningún Estado Contratante podrá, por expulsión o devolución, poner en modo alguno a un refugiado en las fronteras de los territorios donde su vida o su libertad peligre por causa de su raza, religión, nacionalidad, pertenencia a determinado grupo social, o de sus opiniones políticas». De esta manera, el principio resulta aplicable desde el momento en que los solicitantes se encuentran en la frontera y no hayan sido admitidos de manera formal o legal en el territorio, por lo que no pueden ser rechazados o expulsados sin un análisis previo, adecuado e individualizado de su situación (ACNUR, 2007).

Esta protección se otorga a toda persona que sea reconocida como refugiado en los términos de la Convención de 1951, es decir, a cualquier persona que reúna los requisitos de la definición de refugiado consignada en el artículo 1a (2) de la Convención - los denominados criterios de inclusión- y que no se encuentre dentro del ámbito de ninguna de las cláusulas de exclusión. Además, la norma también se aplica a las personas que cumplen con los criterios de elegibilidad establecidos en el artículo 1 de la Convención de 1951, pero cuya condición de refugiado no haya sido formalmente reconocida. Este aspecto tiene particular relevancia para quienes se encuentren en calidad de solicitantes de asilo, puesto que no pueden ser devueltos o expulsados mientras esté pendiente la determinación de su condición, lo que se explica en el carácter declarativo de dicha institución (ACNUR, 1992: par. 28). ${ }^{6}$

núm. 1. Aplicación del artículo 3 en relación con el artículo 22 de la Convención» (CAT/C/GC/4), 4 de septiembre de 2018, disponible en https://bit.ly/38Tjpfk.

5. La Convención sobre el Estatuto Internacional de los Refugiados de 1933 se aplicó principalmente a los refugiados armenios y rusos y fue uno de los principales logros por parte de la Sociedad de Naciones para definir las responsabilidades de los Estados con respecto a las personas que buscaban protección a causa de los desastres de la Primera Guerra Mundial y los diversos conflictos de la posguerra.

6. Así lo ha entendido la Corte Interamericana de Derechos Humanos (Corte IDH), señalando que: «de acuerdo con la Convención de 1951, una persona es un refugiado tan pronto como reúne los requisitos enunciados en la definición, lo que necesariamente ocurre antes de que se determine formalmente su condición de refugiado. Así pues, el reconocimiento de la condición de refugiado de una persona 
El principio de no devolución, tal y como lo dispone el artículo 33 de la Convención de 1951, a su vez forma parte del derecho consuetudinario internacional (Goodwin-Hill, 2014: 5; McBeth, Nolan y Rice, 2018: 508; Corte IDH, 2013: párr. 151; Corte IDH, 2018: párr. 179). Como tal, es vinculante para todos los Estados, incluidos aquellos que no son parte de la Convención de 1951 y/o de su Protocolo de 1967 (ACNUR, 2007: 9). Como resultado, el alcance del principio de no devolución se extiende más allá que lo dispuesto por la Convención de 1951 y ha sido progresivamente incorporado por otros instrumentos de derecho internacional, algunos de los cuales revisaremos en las líneas siguientes.

\section{Principales instrumentos sobre la protección internacional}

\section{La Convención de 1951 y el estatuto de refugiados}

La Convención sobre el Estatuto de Protección de los Refugiados, conocida como la Convención de 1951, surge por la necesidad de brindar protección a las personas que dejaron su país como consecuencia de la Segunda Guerra Mundial. De esta manera, en un primer momento su aplicación quedó acotada temporalmente a los eventos ocurridos antes de 1951 y, geográficamente, a los desplazamientos ocurridos en Europa. Años más tarde, el Protocolo de 1967 eliminó la limitación temporal de la definición de refugiado e hizo extensiva su aplicación más allá de las hipótesis originales. Ambos instrumentos constituyen la piedra angular del régimen de protección internacional de los refugiados.

La definición de refugiado se encuentra contenida en el párrafo 2 de la sección A del artículo 1 de la Convención de 1951 y comprende a toda persona que «debido a fundados temores de ser perseguida por motivos de raza, religión, nacionalidad, pertenencia a determinado grupo social u opiniones políticas, se encuentre fuera del país de su nacionalidad y no pueda o, a causa de dichos temores, no quiera acogerse a la protección de tal país».

En su preámbulo, la Convención señala que las Naciones Unidas han manifestado su profundo interés por los refugiados, y se esfuerzan por asegurarles «el ejercicio más amplio posible de sus derechos y libertades fundamentales». Sin perjuicio de lo anterior, debe notarse que las instituciones e instrumentos de protección internacional están envejeciendo sin poder dar respuesta adecuada a nuevos desafíos (Simeon, 2013: 75 y ss.).

La protección internacional y el concepto de refugiado son dos términos intrínsecamente relacionados (Goodwin-Hill, 2014). En este sentido, los primeros llamados

no tiene carácter constitutivo, sino declarativo» (Corte IDH, Caso Familia Pacheco Tineo vs. Estado Plurinacional de Bolivia. Excepciones Preliminares, Fondo, Reparaciones y Costas, sentencia del 25 de noviembre del 2013, párr. 145, disponible en http://bit.ly/2MlOGAc). 
a recibir protección internacional son aquellos individuos reconocidos como refugiados según lo dispuesto por la Convención de 1951, la que además de esta definición establece criterios de inclusión, exclusión y cesación de esta condición (Reyes Canales y Romero, 2018: 8).

Instrumentos regionales de protección: la Convención de la Organización por la Unidad Africana (OUA) de 1969 y la Declaración de Cartagena de 1984

Existen instrumentos regionales de protección de desplazados y refugiados, sea orientados al refugio o a la protección de derechos humanos (Goodwin-Hill, 2014: 6). Citaremos en primer lugar la Convención de la Organización por la Unidad Africana (OUA) por la que se regulan los aspectos específicos de problemas de los refugiados en África (1969). Durante la década del sesenta hubo numerosos movimientos de refugiados en África. Muchas personas huyeron a países que también eran inestables. Al final de dicha década, más de dos tercios del presupuesto del ACNUR se empleaba en África (ACNUR, 2005a: 9-10). En este contexto, el año 1969 la Organización de la Unidad Africana (actual Unión Africana) adoptó su propia Convención, la que contiene la definición convencional y, al mismo tiempo, desarrolló una definición ampliada de refugiado, considerándolo como «cualquier persona obligada a dejar su país debido a agresión externa, ocupación, dominación extranjera o eventos que perturben gravemente el orden público ya sea en parte o en todo su país de origen o de nacionalidad».

Por su parte, a finales de la década de los setenta y principios de los ochenta, los problemas relacionados con los desplazamientos humanos masivos ocasionados por guerras, conflictos civiles, violencia y agitación política en un número de Estados, especialmente en América Central, dieron origen al contexto en el que surgiría la Declaración de Cartagena sobre Refugiados.7 La Declaración es un instrumento regional de protección adoptado en 1984 por un grupo de expertos de varios países de América Latina como resultado del Coloquio sobre la Protección Internacional de los Refugiados, celebrado en Cartagena de Indias, Colombia. El Coloquio se enfocó en los problemas jurídicos y humanitarios que afectaban a las personas desplazadas en el contexto de crisis humanitarias. A pesar de que la Declaración de Cartagena no es legalmente vinculante, muchos países de América Latina han incorporados sus principios y su definición de refugiado en sus legislaciones y prácticas nacionales.

De esta manera, la definición de refugiado planteada por la Declaración es aquella que además de contener los elementos de la Convención de 1951 y el Protocolo de 1967 , considera como refugiados a «las personas que han huido de sus países porque

7. ACNUR, «La determinación del estatuto de refugiado ¿Cómo identificar quién es un refugiado?» (Módulo autoformativo 2, ACNUR, 1 septiembre 2005), 14. Disponible en https://bit.ly/3rFBLsS. 
su vida, seguridad o libertad han sido amenazadas por la violencia generalizada, la agresión extranjera, los conflictos internos, la violación masiva de los derechos humanos u otras circunstancias que hayan perturbado gravemente el orden público».

\section{Protección complementaria}

El concepto de protección complementaria está plagado de imprecisiones. El término no aparece en ningún tratado internacional y, materialmente, los Estados lo aplican de diversas maneras (Reyes Canales y Romero, 2018: 10 y ss.).

En el año 2005, el Comité Ejecutivo del ACNUR adoptó la primera Conclusión Internacional que trataba específicamente sobre «formas complementarias de protección» (ACNUR, 2005b), sin embargo, no intentó definir el concepto. El Preámbulo del documento enuncia someramente que bajo este concepto se consideran «casos no abordados por la Convención de 1951 y su Protocolo de 1967», incluidas las personas cubiertas por las definiciones ampliadas de refugiado, como las contenidas en la Convención de la Organización para la Unión Africana, la Declaración de Cartagena o la Directiva de Calificación de la Unión Europea.

Por naturaleza, el término protección complementaria es relacional. En otras palabras, esta clase de salvaguarda requiere de la existencia de un régimen formal de protección al que, tal como su nombre sugiere, viene a dar complemento. Por lo tanto, su contenido se determinará y adaptará según el alcance atribuido a esa otra fuente de protección (McAdam, 2007: 23). La protección complementaria tiene tanto un significado legal-formal como uno más genérico. En su acepción puramente descriptiva, comprende una serie de situaciones en las que los Estados han otorgado protección a personas que se encuentran fuera de la definición convencional de refugiado, abarcando conceptos como la protección temporal, la Convención regional de la OUA y la Declaración de Cartagena, entre otros. La palabra complementario en este contexto no se invoca para describir una misma relación jurídica, sino simplemente para referirse a aquella forma de protección que se otorga fuera del instrumento internacional fundamental sobre refugiados.

En su acepción técnico legal, la protección complementaria denota la protección otorgada a las personas sobre una base legal distinta a la Convención de 1951, como podría ser la Convención contra la Tortura de Naciones Unidas, el Pacto Internacional de Derechos Civiles y Políticos, la Convención Americana sobre Derechos humanos, entre otros. En este punto, el desarrollo más amplio del principio de no devolución constituye uno de los principales fundamentos de la protección complementaria, ${ }^{8} \mathrm{y}$

8. Entre los años 1994 y 1995, la Asamblea General aprobó dos resoluciones que reiteraban la importancia de garantizar el acceso para todas las personas que buscan protección internacional, a procedimientos justos y eficientes para determinar el estatuto de refugiado o, a través de otros mecanismos, para 
como tal, será una herramienta de análisis que emplearemos respecto del problema objeto del presente trabajo.

\section{Cambio climático, derechos humanos y desplazamiento forzado}

\section{Sobre el cambio climático}

El Grupo Intergubernamental de Expertos sobre el Cambio Climático (Intergovernmental Panel on Climte Change, IPCC) es una organización internacional, convocada a petición de los estados, establecida por dos agencias de Naciones Unidas, a saber, la Organización Meteorológica Mundial y el Programa de las Naciones Unidas para el Medio Ambiente, y luego refrendado por la Asamblea General de las Naciones Unidas. ${ }^{9}$ Su mandato es facilitar las evaluaciones integrales del estado de los conocimientos científicos, técnicos y socioeconómicos sobre el cambio climático, sus causas, posibles repercusiones y estrategias de respuesta.

En el año 1992, la Organización de Naciones Unidas, a través de la Cumbre para la Tierra celebrada en Río de Janeiro, adoptó la Convención Marco de las Naciones Unidas sobre el Cambio Climático, convirtiéndose en el primer instrumento jurídico internacional con el objeto de reconocer, en su artículo 2, las modificaciones del planeta producto del cambio climático y buscando proteger el sistema climático para las generaciones presentes y futuras con «la estabilización de las concentraciones de gases de efecto invernadero en la atmósfera».

En su artículo 1, este instrumento define el cambio climático como «un cambio de clima atribuido directa o indirectamente a la actividad humana que altera la composición de la atmósfera mundial y que se suma a la variabilidad natural del clima observada durante períodos de tiempo comparables».

Luego, en 1997 fue adoptado el Protocolo de Kioto de la Convención Marco de las Naciones Unidas sobre Cambio Climático. A partir de este instrumento, los Estados parte deben cumplir ciertos compromisos, metas de limitación y reducción de emisiones de dióxido de carbono y determinados gases de efecto invernadero, con el fin de minimizar las repercusiones sociales, ambientales y económicas en dichos países.

Para el cumplimiento de los compromisos indicados por el Protocolo, se estableció un primer período que comprendía de 2008 a 2012. Y el segundo tramo empezó el 1 de enero de 2013, cuya fecha de término será el año 2020.

\footnotetext{
garantizar que las personas necesitadas de protección internacional sean identificadas. Véase ACNUR, «Resolution adopted by the General Assembly A/RES/49/169, 24 de febrero de 1995), disponible en https://www.acnur.org/fileadmin/Documentos/BDL/2006/4575.pdf y ACNUR, «Resolution/ adopted by the General Assembly, A/RES/50/152, 9 de febrero de 1996, disponible en https://www.acnur.org/ fileadmin/Documentos/BDL/2002/1785.pdf.
}

9. Sobre el mismo, véase https://bit.ly/3aURf6D. 
En 2015, dada la preocupación internacional respecto del cumplimiento del Protocolo de Kioto, los Estados parte se reunieron en la Vigésima Primera Conferencia de las Partes en París (COP21), dando vida al Acuerdo de París. En este instrumento internacional el tono de la preocupación varía, de lo que da cuenta al expresar que «el cambio climático representa una amenaza apremiante y con efectos potencialmente irreversibles para las sociedades humanas y el planeta». ${ }^{10}$ Se reconoce, del mismo modo:

El cambio climático es un problema común de la humanidad, [se deben adoptar] medidas para hacer frente, [los Estados deben] respetar, promover y tomar en consideración sus respectivas obligaciones con respecto a los derechos humanos, el derecho a la salud, los derechos de los pueblos indígenas, las comunidades locales, los migrantes, los niños, las personas con discapacidad y las personas en situaciones de vulnerabilidad y el derecho al desarrollo, así como la igualdad de género, el empoderamiento de la mujer y la equidad intergeneracional. ${ }^{11}$

Si bien, el Acuerdo no es un instrumento legalmente vinculante como el protocolo, se enfoca en construir consensos de mayor amplitud, fijar metas por parte de los Estados, especialmente en lo relativo al aumento de compromisos de mitigación y adaptación sobre pérdidas y daños relacionados al cambio climático, desarrollo de tecnologías, fomento de capacidad, entre otros aspectos, para acelerar la aplicación del Convenio y del Protocolo de Kioto, orientando los esfuerzos a limitar el aumento de la temperatura mundial a dos grados Celsius para el año 2020 .

En 2019 estaba prevista la realización de la $25 .^{\circ}$ sesión de la Conferencia de las Partes (COP25) en Chile, en la cual se buscaba fijar criterios para cumplir el Acuerdo de París de 2015 y mejorar las metas de reducción de emisiones de los países partes, lo que se vio frustrado por motivos públicos. En este contexto, aun cuando Chile ha ratificado la Convención Marco, el Protocolo de Kioto y el Acuerdo de París, la legislación interna tiene déficits en cuanto a la regulación del cambio climático. En el año 2017, bajo el mandato de la presidenta Michelle Bachelet, se publicó el Plan de Acción Nacional de Cambio Climático 2017-2022, ${ }^{12}$ en el que se hace una presentación del cambio climático en Chile, la institucionalidad nacional y los avances de nuestro país en la materia (mitigación, adaptación y creación y fomento de capacidades).

En el mismo sentido, una importante crítica es la ausencia de ratificación del Acuerdo Regional sobre el Acceso a la Información, la Participación Pública y el Acceso a la Justicia en Asuntos Ambientales en América Latina y el Caribe (2018) o

10. Acuerdo de París, pp. 2, disponible en https://bit.ly/34REHJl.

11. Ibíd., p. 1.

12. Ministerio del Medio Ambiente, «Plan de Acción Nacional de Cambio Climático 2017-2022», disponible en http://bit.ly/35eoEU8. 
Acuerdo de Escazú. Si bien Chile fue uno de los países más involucrados en el proceso de redacción y negociación de este documento, ${ }^{13}$ el gobierno de Sebastián Piñera se restó de la firma, pese a la existencia de numerosos conflictos socioambientales en el país. ${ }^{14}$

Sin perjuicio de lo anterior, existen algunas iniciativas parlamentarias y gubernamentales con el fin de adecuar la legislación a los desafíos que implica el cambio climático. En lo principal, son las siguientes propuestas: a) Modificación de la Ley de Bases Generales del Medio Ambiente (Ley 19.300) que incorpora el concepto de cambio climático al artículo 2 de la ley en comento, ${ }^{15}$ b) Modificación de nombre del Ministerio del Medio Ambiente a «Ministerio del Medio Ambiente y Cambio Climático»; ${ }^{16}$ y c) Anteproyecto de Ley Marco de Cambio Climático: $:^{17}$ el artículo 1 contemplaría el objeto, expresando que «la presente ley tiene por objeto hacer frente a los desafíos que impone el cambio climático, transitar hacia un desarrollo bajo en emisiones de gases de efecto invernadero hasta alcanzar la neutralidad de emisiones, aumentar la resiliencia a los efectos adversos del cambio climático, y dar cumplimiento a los compromisos internacionales asumidos por el Estado de Chile en la materia». ${ }^{18}$

De este modo, podemos decir que, pese a la adopción de los principales instrumentos internacionales, Chile no ha adecuado su legislación doméstica a los nuevos desafíos que impone el cambio climático, ni tampoco ha abordado la manera en que estas obligaciones se relacionan con aquellas impuestas por los tratados de derechos humanos, con miras a la nueva realidad que se deberá afrontar.

13. Sobre el Acuerdo, véase Claudio Osses Garrido, «Las implicancias para Chile de ratificar el Acuerdo de Escazú», sitio web de la Facultad de Derecho de la Universidad de Chile, disponible en https:// bit.ly/3mAWCKd.

14. Consuelo Contreras, «Columna de opinión: Chile y el Acuerdo de Escazú», INDH, disponible en http://bit.ly/3avlqAY.

15. Cámara de Diputados, Boletín 11.689-12, del 04 de abril de 2018, moción. Estado: Primer trámite constitucional. Este proyecto en su artículo a ter) lo define como: «el cambio identificable del estado del clima, por ejemplo, mediante pruebas estadísticas, en las variaciones de su valor medio y/o en la variabilidad de sus propiedades, que persiste durante períodos de tiempo. El cambio climático puede deberse a procesos internos naturales, a forzamientos externos o a cambios antropógenos persistentes de la composición de la atmósfera».

16. Cámara de Diputados, Boletín 12.509-12 del 2 de abril de 2018, moción. Estado: Primer trámite constitucional. Asimismo, el artículo 3 letra b) entrega una definición legal de cambio climático: «Se entiende un cambio de clima atribuido directa o indirectamente a la actividad humana que altera la composición de la atmósfera mundial y que se suma a la variabilidad natural del clima observada durante períodos de tiempo comparables».

17. Ministerio del Medio Ambiente, "Anteproyecto de Ley Marco de Cambio Climático», disponible en línea en https://bit.ly/3hsJXrX.

18. Para un análisis, véase Espacio Público, «Anteproyecto de ley marco de cambio climático», Recomendación Legislativa núm. 25, Santiago, 2018, disponible en https://bit.ly/305sTuG. 


\section{Derechos humanos, medio ambiente y cambio climático}

La literatura especializada ha destacado que los derechos humanos ligados al medio ambiente, originalmente restringidos a la Carta Africana de Derechos Humanos y de los Pueblos (1981), están ganando mayor reconocimiento internacional general (Smith, 2016: 412). Si bien, a la fecha no existe un instrumento de sistema universal que garantice el derecho al medio ambiente, algunos creen posible una lectura en este sentido a partir del artículo 25.1 de la Declaración Universal de los Derechos Humanos: ${ }^{19}$ "Toda persona tiene derecho a un nivel de vida adecuado que le asegure, así como a su familia, la salud y el bienestar».

En relación con lo anterior, no es sorpresivo afirmar que el cambio climático afectará el disfrute y goce de diversos derechos humanos alrededor del mundo, sea por la erosión costera, inundaciones, sequías, lluvias, aumento del nivel del mar, en particular en el caso de Estados insulares del Pacífico, entre otros efectos proyectados. Sobre esto, en junio de 2019, el Relator Especial sobre la Extrema Pobreza de la ONU, Philip Alston, sostuvo que el cambio climático y su aumento de dos grados Celsius podría provocar que entre uno y dos billones de personas no tengan acceso adecuado al agua. Y que, entre 2030 a 2050 se espera que mueran adicionalmente 250.000 personas por año debido a la desnutrición, malaria, diarrea y estrés por calor. Solo en el año 2017,18 ,8 millones de personas se desplazaron ${ }^{20}$ producto de las transformaciones medioambientales que suscitan su expresión por medio del cambio climático.

En la misma línea, la Alta Comisionada de las Naciones Unidas para los Derechos Humanos, Michelle Bachelet, señaló recientemente que «la crisis climática se está acelerando [y que el] calentamiento global ya afecta los derechos humanos de millones de personas expuestas a sus efectos adversos cada año. Las personas de las naciones isleñas se encuentran entre las más inmediatamente afectadas, con un impacto diario en el disfrute colectivo de sus derechos al agua y su saneamiento, la salud, la educación, la alimentación, el trabajo, la vivienda adecuada y el derecho a la libre determinación». ${ }^{21}$ En concordancia con lo anterior, el Relator Especial sobre los Derechos Humanos y el Medio Ambiente, David R. Boy, previamente había solicitado que se reconociese formalmente el derecho humano al aire limpio, al agua potable, a los alimentos sanos, a un clima estable, a una biodiversidad próspera y a ecosistemas

19. Desde luego, implica hacerse cargo sobre el estatus de la Declaración Universal y su fuerza normativa. Sobre su fuerza normativa, véase Villán Durán (1998: 73-89).

20. Organización Internacional de las Migraciones (OIM), «Migración y cambio climático», disponible en http://bit.ly/3aTwEzx; OIM, «Climate Change and Migration in Vulnerable Countries. A snapshot of least developed countries, landlocked developing countries and small island developing States», disponible en http://bit.ly/2J7Ji2g.

21. ACNUDH, «Human Rights and Climate Change Conference, Nadi, Fiji, August 2019. Michelle Bachelet, UN High Commissioner for Human Rights», disponible en http://bit.ly/3asGAQl. 
saludables ${ }^{22}$ para combatir los efectos del cambio climático, ya que el derecho a un medio ambiente sano posee protección constitucional en más de cien países. En el informe ${ }^{23}$ de marzo 2019 se dio cuenta sobre las consecuencias adversas del cambio climático en relación con las obligaciones de derechos humanos relacionadas con el disfrute de un medio ambiente seguro, limpio, saludable y sostenible, a saber:

- contaminación ambiental y doméstica;

- impactos en la salud humana;

- impactos en población vulnerable;

- impactos en la agricultura, biodiversidad y ecosistemas;

- relación entre contaminación del aire y cambio climático; y

- derecho a la salud.

Concluye instando en forma urgente a la toma de medidas concentradas y sistemáticas, con un enfoque prioritario en las personas más vulnerables. Hace un especial llamado a efectuar un cambio rápido de los combustibles fósiles a energías renovables, ya que tal transición podría salvar la vida de 150 millones de personas durante el siglo XXI.

Por tanto, existe plena conciencia de la directa y estrecha relación entre derechos humanos y el cambio climático. Los efectos tan nocivos, presentes y futuros de este repercuten directamente en la vida, la libertad, la salud, la educación y el entorno, afectando de forma más directa a la población económica y socialmente vulnerable. Pero, a fin de cuentas, perturba de forma global a todos los habitantes del planeta y a las futuras generaciones.

\section{Cambio climático como hipótesis de protección internacional}

Hemos revisado hasta ahora que los efectos del cambio climático amenazan con exponer y profundizar la vulneración de los derechos de grupos humanos completos. No obstante, el sistema internacional de protección de derechos humanos pareciera no considerar, al menos explícitamente, causales de protección relativas a los efectos del cambio climático y los desastres que provocará.

Desde esta perspectiva, es importante considerar que, aunque el derecho internacional define refugiado de una manera particular, esto no debe llevarnos a concluir

\footnotetext{
22. Naciones Unidas, «El derecho humano a un medio ambiente sano debe ser reconocido», disponible en https://news.un.org/es/story/2018/10/1444342.

23. John Knox, «Issue of human rights obligations relating to the enjoyment of a safe, clean, healthy and sustainable environment: Report of the Special Rapporteur», disponible en https://bit.ly/34P1oPZ.
} 
apresuradamente que quienes están fuera de la definición, no puedan recibir algún tipo de protección jurídica si igualmente lo requieren. Debe privilegiarse una interpretación coherente con lo anterior, sobre todo, considerando que el fin último de la protección internacional es garantizar el resguardo hacia las personas frente a vulneraciones graves a sus derechos básicos.

A continuación, analizaremos brevemente las distintas posibilidades de otorgar protección a migrantes por causas climáticas según los instrumentos de protección vigentes sobre refugio en el sistema universal de derechos humanos, en los sistemas regionales y, finalmente, revisaremos lo relativo a protección complementaria.

\section{Aplicación de la Convención de 1951 y los instrumentos universales de protección}

La definición legal de refugiado y los derechos que esta calidad conlleva, se establecen en la Convención de 1951 sobre el Estatuto de los Refugiados, junto al Protocolo de 1967. Como se señaló, un refugiado se define como quien «debido al temor fundado de ser perseguido por motivos de raza, religión, nacionalidad, pertenencia a un grupo social particular u opinión política, está fuera del país de su nacionalidad y no puede, o debido a tal temor, no está dispuesto a valerse de la protección de ese país».

Pues bien, al examinar si el desplazamiento por motivos climáticos puede comprenderse dentro de esta definición, lo cierto es que una negativa salta a la vista. Primero, la definición de refugiado únicamente se aplica a personas que ya han cruzado una frontera internacional. Como se planteó, el desplazamiento en respuesta al cambio climático mayoritariamente será interno y, por lo tanto, no cumplirá este requisito. En segundo lugar, existen dificultades para caracterizar el cambio climático como persecución, pues asumirlo como tal nos lleva inmediatamente a preguntarnos quién sería el agente persecutor. ${ }^{24}$ Aunque han existido argumentos para justificar en este sentido, resultan bastante forzados, pues no existe una política persecutoria (Cooper, 1998: 480; Kozoll, 2004: 271). Además, el elemento persecución debe encontrarse vinculado al menos a una de las cinco causales enunciadas en la Convención, las que responden a características particulares, como los antecedentes o creencias de una persona, es decir, raza, religión, nacionalidad, pertenencia a un determinado grupo social u opiniones políticas (ACNUR, 1992: 66-86). La dificultad, en el contex-

24. El Tribunal de Revisión de Refugiados de Australia (RRT) ha rechazado el argumento de que los daños a causa de un desastre climático equivaldrían a «persecución» para los efectos de la Convención de Refugiados. Simplemente no hay base para concluir que los países que históricamente han sido emisores altos de dióxido de carbono u otros gases de efecto invernadero tienen algún elemento de motivación para tener un impacto en los residentes. Véase Tribunal de Refugiados para Australia, RT Case 0907346, [2009] RRTA 1168, Australia: Refugee Review Tribunal, 10 de diciembre de 2009, disponible en http://bit.ly/2KP 7 mHZ 
to actual, es que los impactos del cambio climático son en gran medida indiscriminados, y afectan incluso a la propia población de los países que más contribuyen a la contaminación ambiental.

De esta manera, podemos sostener que el desplazamiento forzado por razones climáticas difícilmente podría enmarcase dentro de los límites actualmente reconocidos por la Convención, los que se corresponden con la idea tradicional de persecución (Storey, 2014: 272). ${ }^{25}$

\section{Aplicación de los instrumentos regionales de protección}

Tal como revisamos, la Convención Regional de la Unión Africana y la Declaración de Cartagena en América Latina contienen definiciones de refugiado más amplias que aquella de la Convención de 1951. La Convención africana incluye como refugiados a personas desplazadas debido a una serie de causales a las que nos referimos con antelación. Una de ellas corresponde a «eventos gravemente inquietantes al orden público», y se ha planteado si en virtud de esta podrían abarcarse los efectos dejados tras una catástrofe ambiental, como la hambruna, tormentas, alza en las temperaturas y la sequía.

Parte de la doctrina ha argumentado que tal interpretación es teóricamente posible (Edwards, 2006: 225-227; McAdam, 2007; Kälin, 2010: 88-89), ${ }^{26}$ pero señala que a pesar de que las personas que huyen de tales catástrofes «con frecuencia se refugian en el territorio de los Estados vecinos, los Estados receptores rara vez declaran que están actuando de conformidad con las obligaciones de la Convención de la OUA» (McAdam, 2007: 89). La práctica general de acoger a personas desplazadas por eventos ambientales, en este sentido, «puede verse como una contribución al desarrollo de un derecho de protección temporal por razones humanitarias en virtud del derecho internacional consuetudinario, en lugar de en virtud de un tratado» (Edwards, 2006: 227).

Análogamente, la Declaración de Cartagena considera una causal similar, que extiende la protección en caso de «circunstancias que han perturbado gravemente el orden público». Si bien la noción de orden público no tiene una definición universalmente aceptada, puede ser interpretada en el contexto de la acepción de refugiado de la Declaración de Cartagena como una referencia a la paz y la seguridad de la sociedad y el funcionamiento normal de las instituciones del Estado con pleno respeto a

25. Cabe hacer presente que la definición de persecución en otros instrumentos internacionales es mucho más amplia, por ejemplo, en el artículo 7(1)(h) en relación con el artículo 7(2)(g) del Estatuto de Roma en el ámbito del derecho penal internacional.

26. Hathaway (1991: 16-21) argumenta que la definición no sugiere que las víctimas de desastres naturales o mala fortuna económica deben ser responsabilidad de la comunidad internacional. En el mismo sentido, véase Schwartz (1993: 355-380). 
los derechos humanos. De esta manera, las perturbaciones al orden público han sido concebidas como resultado de actos del ser humano y no de desastres naturales. Este concepto incluye disturbios y tensiones internas, como motines, actos de violencia aislados y esporádicos y otros actos de naturaleza similar, ${ }^{27}$ apartándose con ello de causales de protección que pudieran vincularse a desastres naturales. Con todo, es posible que, a consecuencia más o menos directa del cambio climático, se generen hechos de violencia y/o una respuesta de represión violenta por parte de los Estados, lo cual indubitadamente podría encuadrarse en esta causal.

\section{Aplicación de la protección complementaria}

La afectación de derechos humanos como resultado del cambio climático tiene una serie de consecuencias prácticas. En lo que a la protección internacional se refiere, el derecho internacional de los derechos humanos puede proporcionar una base legal sobre la cual se puede buscar - y otorgar- protección en otro Estado, al mismo tiempo que exige la observancia de normas mínimas de tratamiento en el Estado de acogida y, por lo tanto, es relevante para la situación jurídica de los desplazados (McAdam, 2012: 55).

El derecho internacional de los derechos humanos ha ampliado las obligaciones de protección de los Estados más allá de la categoría de refugiado, para incluir personas en riesgo de privación arbitraria de la vida, tortura o tratos o penas crueles, inhumanas o degradantes. Esto se conoce como protección complementaria porque describe la protección basada en los derechos humanos más allá de los deberes que impone la Convención de 1951 y se encuentra basada fundamentalmente en el principio de no devolución.

Ahora bien, no cualquier violación de los derechos humanos puede dar lugar a una obligación de no devolución. Al observar una de las fuentes de la protección complementaria - que examinamos en el primer acápite-, correspondiente al artículo 3 de la Convención contra la Tortura y otros Tratos o Penas Crueles, Inhumanas o Degradantes, podemos analizar cómo el desarrollo jurisprudencial ha circunscrito cuidadosamente el significado de trato inhumano o degradante para que no pueda usarse como remedio para la pobreza general, el desempleo o la falta de recursos o atención médica, excepto en las circunstancias más excepcionales (McAdam, 2012: 9-10).

27. Conferencia Internacional sobre Refugiados Centroamericanos (CIREFCA), Evaluación de la puesta en práctica de las disposiciones del Documento «Principios y criterios para la protección y asistencia a los refugiados, repatriados y desplazados Centroamericanos en América Latina» CIREFCA/ REF/94/1, 28 de junio de 1994, párr, 33. 
La jurisprudencia existente no ha considerado que los impactos del cambio climático constituyan un trato inhumano, principalmente porque dicha noción va ligada a la acción humana directa e identificable, y no se refiere a aquellos efectos mediatos y provenientes de una multiplicidad de fuentes. Para esto, sería necesario un desarrollo sustancial de dicho concepto y de la relación de la acción humana con el concepto, por ejemplo, mediante el establecimiento de deberes de cuidado específicos de un Estado en relación con el cambio climático y sus consecuencias.

A nuestro entender, los derechos humanos clave a considerar en el contexto de protección complementaria en casos de desplazamiento por motivos climático son: a) el derecho a la vida; $y$ b) el derecho a no ser sometido a tortura ni a tratos o penas crueles, inhumanas o degradantes. Si bien estos derechos no son los únicos que conllevan una obligación de no devolución, respecto de ellos no existe discusión en el derecho internacional, en cuanto a que originan dicha obligación. Existen otros casos indubitados, pero que no son pertinentes, como el derecho a no ser sometido a una desaparición forzada. ${ }^{28} \mathrm{~A}$ continuación, desarrollaremos brevemente cada uno de ellos en el contexto de desplazamiento por razones climáticas.

\section{El derecho a la vida y su protección}

El derecho a la vida se encuentra reconocido en el artículo 3 de la Declaración Universal de Derechos Humanos, en el artículo 6 del Pacto Internacional de Derechos Civiles y Políticos, en el artículo 6 de la Convención sobre los Derechos del Niño, y en todos los tratados regionales de derechos humanos, por ejemplo, en el artículo 2 del Convenio Europeo de Derechos Humanos, en el artículo 4 de la Convención Americana sobre Derechos Humanos, y en el artículo 4 de la Carta de Banjul. Adicionalmente, ha sido descrito por el Comité de Derechos Humanos de las Naciones Unidas como el derecho supremo que es básico para todos los derechos humanos. ${ }^{29}$ No es derogable y está reconocido como uno de aquellos cuya amenaza genera el deber de no devolución.

El derecho a la vida está estrechamente relacionado con otros derechos humanos, tanto civiles y políticos, como económicos y sociales..$^{30}$ Se ha argumentado que el de-

28. En este sentido, véase el artículo 69 de la Convención Internacional para la protección de todas las personas contra las desapariciones forzadas.

29. Comité de Derechos Humanos, «Observación General núm. 36 sobre el artículo 6 del Pacto Internacional de Derechos Civiles y Políticos, relativo al derecho a la vida», CCPR/C/GC/R.36/Rev.7, del 3 de diciembre de 2019, párr. 2.

30. Entre otros, aquellos garantizados por el Pacto Internacional de Derechos Económicos, Sociales y Culturales, como la protección y asistencia a la familia (artículo 10), derecho a un nivel de vida adecuado (artículo 11), derecho al disfrute del más alto nivel posible de salud (artículo 12). Sobre las obligaciones jurídicas y el compromiso de garantía de estos derechos sin discriminación, véase Comité de Derechos 
recho a un nivel de vida adecuado -incluidos alimentos, ropa, vivienda y la mejora continua de las condiciones de vida - y el derecho a no ser privado de un medio de subsistencia son componentes necesarios de este derecho. La vida se ve comprometida cuando el calentamiento global conduce, entre otros, a la destrucción de la capacidad de las personas para cazar, pescar, recolectar o emprender la agricultura de subsistencia, a la destrucción de ecosistemas, entre otros (McAdam 2012: 56). ${ }^{31}$

\section{El derecho a no ser sometido a tortura ni a tratos o penas crueles, inhumanas o degradantes}

La Convención contra la Tortura y otros Tratos o Penas Crueles, Inhumanas o Degradantes y el Pacto Internacional de Derechos Civiles y Políticos, así como otros instrumentos regionales de protección, consagran de manera expresa la prohibición de la tortura y los tratos o penas crueles, inhumanos o degradantes sobre la persona en tanto a su protección jurídica en un marco fundamental. La prohibición de la tortura se considera en el mismo sentido como norma de ius cogens (Sferrazza y Bustos, 2019: 148-149).

El enfoque estándar del Comité de Derechos Humanos de la ONU es considerar que estas formas de malos tratos caen en una escala móvil o de jerarquía; la tortura es la manifestación más severa. ${ }^{32}$ La distinción entre tortura y trato inhumano es de grado. El Comité de Derechos Humanos de la ONU considera que no es deseable establecer un concepto cerrado de estos términos: «[e]l Pacto no contiene definición alguna de los conceptos abarcados por el artículo 7, ni tampoco el Comité considera necesario establecer una lista de los actos prohibidos o establecer distinciones concretas entre las diferentes formas de castigo o de trato; las distinciones dependen de la índole, el propósito y la severidad del trato aplicado». ${ }^{33}$

Económicos Sociales y Culturales, Observación General núm. 3: La índole de las obligaciones de los Estados partes (pár. 1 del art. 2 del Pacto). E/1991/23, del 14 de diciembre de 1990, párr. 1; Bou Franch y Castillo Daudí (2010: 80 y ss.).

31. El Comité de Derechos Humanos ha observado que el derecho «abarca la existencia en dignidad humana con las necesidades mínimas de la vida». Del mismo modo, la Convención sobre los Derechos del Niño vincula el derecho a la vida con el deber de los Estados de garantizar en la mayor medida posible la supervivencia y el desarrollo del niño. El Comité de los Derechos del Niño ha explicado la necesidad de ver e implementar el derecho a la vida de manera integral, «a través de la aplicación de todas las demás disposiciones de la Convención, incluidos los derechos a la salud, la nutrición adecuada, la seguridad social, un nivel de vida adecuado, [y] un ambiente sano y seguro». Esto también se refleja en las Directrices Operativas del Comité Permanente entre Organismos (IASC) sobre la protección de las personas en situaciones de desastres naturales.

32. Sobre la gravedad, Villán Durán (1985): 388-389.

33. Comité de Derechos Humanos. Observación General No 20. Reemplaza a la Observación General 
Las lecciones aprendidas de los grandes desastres naturales registrados en la última década han abarcado una pluralidad de medidas esencialmente pragmáticas que tienen como objetivo prevenir la violación o restablecer el ejercicio de derechos fundamentales que pudieron verse afectados a consecuencia del desastre. Entre ellas, evitar el retorno a una situación que podría constituirse en una tortura, un trato inhumano, cruel o degradante, es una hipótesis que no debiera descartarse.

En el caso de la Convención contra la Tortura y otros Tratos o Penas Crueles, Inhumanas o Degradantes, consagra en su artículo 3 la prohibición de devolución, en los siguientes términos:

1. Ningún Estado parte procederá a la expulsión, devolución o extradición de una persona a otro Estado cuando haya razones fundadas para creer que estaría en peligro de ser sometida a tortura. 2. A los efectos de determinar si existen esas razones, las autoridades competentes tendrán en cuenta todas las consideraciones pertinentes, inclusive, cuando proceda, la existencia en el Estado de que se trate de un cuadro persistente de violaciones manifiestas, patentes o masivas de los derechos humanos.

A partir del análisis, se desprende que la prohibición de devolución a la que hace referencia este instrumento es de carácter absoluto e inderogable y, por lo tanto, aplicable a toda persona independiente de su conducta, nacionalidad o circunstancia; no permite excepción alguna a la norma, debido a que la prohibición contra la tortura se considera una norma imperativa de derecho internacional, y diferencia de la aplicación del artículo 33 de la Convención de 1951 la que, tal como se explicó anteriormente, permite excepciones al principio de no devolución recogido en su artículo 33.2. A través de la aplicación de esta norma, puede subsanarse la indefensión a la que se ven sometidas las personas que solicitan protección alegando una persecución que no se encuentre vinculada con alguno de los cinco motivos contenidos en su artículo 1, pero en cuyo caso, sin embargo, la devolución pudiese traer aparejada el sometimiento a torturas o a condiciones que puedan constituir tratos crueles, inhumanos o degradantes.

Cabe señalar, que el ACNUR ha acogido positivamente la aplicación del artículo 3 del Convención contra la Tortura como una nueva vía de protección, lo que ha manifestado en los siguientes términos: «En la práctica, este desarrollo complementario del principio de no devolución está agregando una nueva vía de protección para las personas rechazadas erróneamente por el estatus de refugiado o con claras necesidades de protección, y cuyas circunstancias, sin embargo, no se abordan directamente en la Convención de 1951» (ACNUR, 1998).

7, prohibición de la tortura y los tratos o penas crueles (art. 7), 44 Período de Sesiones, 1992, disponible en https://bit.ly/2L4FnnI. 
Por su parte, el Pacto Internacional de Derechos Civiles y Políticos en su artículo 7 está redactado en términos más amplios, disponiendo que «nadie será sometido a torturas ni a penas o tratos crueles, inhumanos o degradantes». La importancia de este enunciado radica en su alcance, dado su carácter absoluto según el derecho internacional, que permitiría fundar la protección respecto de otras circunstancias que puedan ser caracterizadas también como tratos inhumanos o degradantes. A través de este instrumento, se encuentra protegida cualquier persona, incluidas aquellas que hayan sido solicitantes de asilo, como puede ser un migrante climático, y cuyo reconocimiento de la condición de refugiado se hubiere rechazado. De esta manera, el PIDCP en conexión con la Convención contra la Tortura protege a las personas contra la devolución, en la medida que se vean expuestas a cualquier daño irreparable, como la privación de la vida - protegida conforme el artículo 6 del Pacto-, o la tortura, un trato cruel, inhumano o degradante -artículo 7-, ya sea en el país en que se pretende llevar a cabo la expulsión o en cualquier otro país del cual la persona vaya a ser posteriormente expulsada. ${ }^{34}$

Si bien la Convención contra la Tortura exige una finalidad, en los hechos lo que aquí se requiere es configurar que la expulsión de una persona a un lugar afectado por condiciones de este tipo pueda configurar un trato degradante y pueda causar dolores graves de tipo físico o psíquico. Con todo, este tema amerita un estudio más profundo.

\section{Viabilidad en la creación de un nuevo instrumento internacional}

A lo largo de las líneas anteriores, hemos revisado distintas posibilidades de otorgar protección internacional a quienes se han desplazado por motivos climáticos, a la luz del marco normativo vigente. Sin embargo, antes de terminar este ensayo, no podemos dejar de mencionar una moción que se ha levantado desde diversos sectores para otorgar protección internacional en esta hipótesis, pues hoy existe una brecha a salvar, sea por medio de ampliar la definición de persecución, o por la vía de declarar que los refugiados ambientales pertenecen a un grupo social, o por diseñar un nuevo instrumento. ${ }^{35}$

Esto significaría la creación o modificación del marco legal internacional vigente en favor de los migrantes climáticos, buscando acuñar el concepto de refugiados climáticos. Las propuestas incluyen desde la creación de un protocolo a la Convención

34. Cfr. Comité de Derechos Humanos, Observación General núm. 31. Naturaleza de la obligación jurídica general impuesta a los Estados Parte en el Pacto, CCPR/C/21/Rev.1/Add.13., 26 de mayo de 2004, párr. 12.

35. Sobre esta brecha, y algunas alternativas no exhaustivas, véase: Addressing the Protection Gap of Environmental Refugees: A Reform of the 1951 Refugee Convention?, Disponible en http://bit. ly/3axPDzo 
de Refugiados hasta un tratado independiente basado en los principios de solidari$\operatorname{dad}^{36} \mathrm{y}$ responsabilidad internacional. ${ }^{37}$

Sin embargo, dentro del panorama político e internacional actual,,$^{38}$ pareciera que una alternativa de este tipo no resulta viable. En este sentido, el ACNUR ha venido trabajando estos problemas desde mediados de la década pasada, y prestando asistencia técnica en estas materias. ${ }^{39}$ Esto se debe en parte a los intereses incompatibles de los posibles países de origen y los países de destino de tales movimientos. Mientras que los primeros probablemente presionarían por un máximo de derechos con respecto a la admisión y el estatus, los segundos, teniendo en cuenta su actitud restrictiva hacia los refugiados y los solicitantes de asilo, no es probable que acepten más que las obligaciones contraídas en una época mucho más propicia a considerar la situación de los refugiados producto de la experiencia de la Segunda Guerra Mundial.

De este modo, parece ser que este abordaje tendrá que esperar todavía, de modo que no podemos mencionarlo como una solución para casos concretos. Así las cosas, es aquí donde radica la importancia de la reconstrucción normativa ofrecida anteriormente, en tanto ya existen en el marco jurídico internacional otros instrumentos que pueden servir a estos efectos.

36. La solidaridad internacional aparece como un principio aplicable en los hechos a los países que reciben enormes flujos de refugiadas y refugiados, escapando al control que cada Estado puede ejercer sobre ellos, saturando su capacidad para brindar la protección y asistencia que requieren esas personas en virtud de las convenciones señaladas. Ya en 1998, el Comité Ejecutivo de ACNUR se pronunciaba respecto de una de las alternativas para enfrentar estas circunstancias en la Conclusión General sobre la Protección Internacional 85: «El Comité Ejecutivo reconoce que la solidaridad y el reparto de la carga entre los países tienen importancia directa para la aplicación satisfactoria de los principios de protección de los refugiados, si bien subraya que la obtención de asilo y el cumplimiento por los Estados de sus obligaciones en materia de protección no deben depender de que previamente se adopten disposiciones y acuerdos en materia de reparto de la carga, en particular porque el respeto de los derechos humanos fundamentales y de los principios humanitarios es obligación de todos los miembros de la comunidad internacional», disponible en https://bit.ly/3rFHE9E.

37. Si los Estados son legal y fácticamente responsables del cambio climático, y en qué medida lo son, es uno de los temas más complicados y debatidos que se han examinado. En el derecho ambiental internacional, la atmósfera global y el clima se consideran un recurso común de vital interés para la humanidad.

38. Estas reflexiones fueron escritas antes de la pandemia que actualmente azota al mundo, pero desde luego, la conclusión puede mantenerse, especialmente considerando el autoritarismo de ciertos regímenes que han aprovechado la situación de salud para imponer restricciones y limitar el acceso a derechos humanos.

39. ACNUR, «Why UNHCR is taking action on climate change displacement», disponible en http:// bit.ly/34WOjlW. 
Observaciones preliminares sobre indicadores de análisis para la concesión de protección internacional por motivos climáticos

A modo de síntesis de los argumentos aquí expuestos, es de vital importancia establecer cuál o cuáles aspectos deben evaluarse en el caso de que una persona solicite protección internacional sobre la base del daño que ha sufrido o su retorno pudiera ocasionarle por motivos vinculados al cambio climático, y la forma en que eso podría ser abordado. De esta manera, los requisitos o indicadores que debieran ser considerados para poder argumentar una solicitud de estas características en forma plausible ante un órgano tomador de decisiones administrativo o jurisdiccional, son los siguientes:

El daño, la afectación o amenaza alegado por la persona o el grupo solicitante de protección internacional debe vincularse directa o indirectamente a razones derivadas del cambio climático.

El umbral de ese daño, afectación o amenaza a los derechos de la persona solicitante debe ser grave y referirse especialmente al derecho a la vida, integridad física y psíquica, seguridad, protección de la salud, entre otros.

A la luz de la entidad del daño, afectación y/o amenaza, y la entidad de los derechos afectados, se debe argumentar que el retorno o refoulment de la persona a ese contexto pudiera poner en peligro la vida de la persona, constituir una situación análoga a una tortura o trato cruel, inhumano o degradante.

Los principales tratados han sido revisados a lo largo de este artículo, y aunque se reconoce que pudieran existir otros instrumentos que podrían citarse, lo cierto, es que estos instrumentos del sistema universal de protección de derechos humanos gozan de una amplia ratificación por los Estados y han sido frecuentemente citados como fuentes protección complementaria.

De este modo, los indicadores citados, sustentados en los instrumentos internacionales que acaban de indicarse, constituyen una primera aproximación a la construcción del fundamento necesario para brindar protección internacional, de acuerdo con las obligaciones suscritas por los Estados, de modo que puedan ser defendidos en distintos sistemas jurídicos para afrontar la problemática del desplazamiento forzado por motivos de desastre o cambio climático.

En el sistema chileno debieran considerarse especialmente las obligaciones contenidas en el Pacto Internacional de Derechos Civiles y Políticos, la Convención contra la Tortura y otros Tratos o Penas Crueles, Inhumanas o Degradantes, la Convención Interamericana para Prevenir y Sancionar la Tortura, la Convención Americana de Derechos Humanos, además de tratados como la Convención sobre los Derechos del Niño, la Convención sobre la Eliminación de todas las Formas de Discriminación contra la Mujer, y la Convención Interamericana para Prevenir, Sancionar y Erradicar la Violencia contra la Mujer o Convención de Belém do Pará, entre otros. 
Estimamos, por su parte, que la vía más idónea para poder resguardar esto, en caso de una respuesta negativa de protección por parte de la Administración del Estado, son las acciones constitucionales de amparo y protección. Esbozaremos los principales argumentos respecto de cada una.

La acción de amparo del artículo 21 de la Constitución Política de la República de Chile, en relación con sus artículos 5 inciso segundo, 19 número 7 y 26, en conexión con los artículos 1.1, 2, 7, 8 y 25 de la Convención Americana sobre Derechos Humanos y los artículos 2.1, 9 y 14 del Pacto Internacional de Derechos Civiles y Políticos, permite salvaguardar la libertad personal y la seguridad individual, ${ }^{40}$ en particular si existe peligro de una expulsión de regreso a un territorio afectado por un desastre climático. Esta acción constituye una garantía rápida y eficaz para defender estos derechos en situaciones de urgencia, así como permitir hipótesis tanto preventivas como de remedio. Por último, la judicatura, conociendo de estas acciones constitucionales, puede disponer las medidas más amplias para restablecer el imperio del derecho y asegurar la debida protección del afectado.

En materia de acción de protección del artículo 20 de la Constitución chilena, conforme a los artículos 5 inciso segundo, 19 número 1 y 26 de la misma, y en relación con las normas citadas de la Convención Americana sobre Derechos Humanos incluyendo los artículos 4, sobre el derecho a la vida, y 5, sobre el derecho a la integridad personal, y del Pacto Internacional sobre Derechos Civiles y Políticos, incluyendo su artículo 4, el recurso de protección también considera hipótesis de preventivas como reactivas, y permite amparar un rango mucho más amplio de derechos protegidos. ${ }^{41}$ Con todo, su tramitación es mucho más lenta que aquella del amparo.

\section{Conclusiones}

Los impactos del cambio climático ciertamente incidirán en el aumento del desplazamiento de miles de seres humanos en distintas regiones del mundo. La evidencia empírica sugiere que la mayoría de estos movimientos se producirán dentro de los países. Por otro lado, la experiencia sugiere que estos desplazamientos tendrán lugar a una velocidad más lenta en comparación a los movimientos motivados por conflictos desencadenados por el hombre. En otras palabras, la aparición lenta de algunos impactos del cambio climático, como el aumento del nivel del mar,o de las temperaturas, brindan una oportunidad única para planificar respuestas. Sin embargo, tras reconocer estas particularidades, resulta fundamental apreciar la forma en que el cambio climático se relaciona e interactúa con las presiones políticas, económicas, sociales y ambientales subyacentes.

40. Sobre la libertad personal, véase Nogueira Alcalá (2002: 170) y Henríquez (2019: 12-14).

41. Sobre esto, véase García y Contreras (2014: 50-52) y Henríquez (2019: 35-36). 
El cambio climático se concibe mejor como un multiplicador de amenazas que exacerba las vulnerabilidades preexistentes. De esta manera, constituye un punto de inflexión respecto del cual deben existir respuestas a la altura por parte de la comunidad de Estados. En caso contrario, el salvarse o no dependerá de las propias personas afectadas, de umbrales de tolerancia individual, recursos, mecanismos de apoyo y el alcance de las redes familiares y de parentesco en otros lugares para ayudar a facilitar el movimiento y proporcionar asistencia a la llegada. Decidir si moverse o no es, por tanto, un proceso complejo.

El derecho internacional de los refugiados es un marco engorroso para abordar la huida por motivos relacionados con el cambio climático. Fue ideado para un contexto diferente, y en la mayoría de los casos será un vehículo inapropiado para responder al desplazamiento ambiental. La primera de estas razones se debe a que, en caso de movimientos mayoritariamente internos, no opera el derecho internacional de los refugiados. Al mismo tiempo, resulta difícil configurar el elemento persecución y más aún el agente persecutor en el sentido convencional. Por su parte, en lo relativo a la aplicación de instrumentos regionales como la Convención Americana y la Declaración de Cartagena, la única causal que pudiera entenderse en términos más amplios a la hora de comprender los efectos del cambio climático es la relativa al orden público, presente en ambos instrumentos. No obstante, hasta el momento, esta hipótesis solo ha sido interpretada en relación con la estabilidad político-social; sin perjuicio del malestar civil, las protestas y la subsecuente represión que podría venir aparejada en el evento de un mal manejo de la crisis.

Ante este escenario, lo más apropiado parecería ser la creación de un nuevo instrumento internacional que regule claramente las hipótesis relacionadas con el desplazamiento forzado a causa de razones ambientales. Sin embargo, al día de hoy, con un auge de gobiernos populistas y que ganan réditos por criminalizar la migración, una alternativa de esta naturaleza no resulta realista. Es allí donde la protección complementaria pudiera tomar un rol protagónico, contribuyendo a sustentar jurídicamente la siguiente premisa: la devolución de una persona, o un grupo de personas, por parte de un Estado, a un lugar que fue duramente afectado por el cambio climático (del cual fuera originara o correspondiera a su lugar de residencia habitual), en condiciones tales que este retorno implicase la afectación grave de sus derechos comprometiendo su integridad y posibilidades de supervivencia, resultaría una forma de violación de la obligación de no devolución (non refoulment).

Estos instrumentos en los que basamos nuestra propuesta, cuentan con una amplia ratificación en la comunidad internacional. En apoyo de esta conclusión, podemos señalar que el principio de no devolución es considerado como una norma de derecho internacional consuetudinario, por lo que su aplicación sería procedente aun cuando un Estado no haya ratificado el instrumento. 
No existe un listado taxativo de violaciones a los derechos humanos que den origen a la obligación de no devolución y es precisamente sobre este punto donde radica la flexibilidad de su aplicación. Ahora bien, sostenemos que es plausible argumentar esto en el caso de la migración causada por el cambio climático basándonos en el derecho a la vida y el derecho a no ser sometido a tortura o tratos crueles inhumanos o degradantes, como detonantes de esta obligación. Por lo tanto, la tarea del responsable de la toma de decisiones es determinar si devolver al individuo en particular a las condiciones generales del país de origen equivaldrá a una violación de uno de estos derechos y los estándares que deberán ser satisfechos. La forma de reclamar esto en cada sistema jurídico será diverso, pero en el sistema jurídico chileno nos parece que las acciones constitucionales de protección y amparo pueden ser la vía para tutelar estas afectaciones, aun cuando no estén comprendidas dentro de las hipótesis para ser considerado refugiado.

Así, y ante la ausencia de normas que regule de manera clara esta materia, con este trabajo esperamos ofrecer una reconstrucción sobre la forma en que podría fundarse una interpretación expansiva de la protección complementaria, basada en fuentes de derecho internacional, convirtiéndose, de esta manera, en una herramienta que contribuya a construir una mirada más amplia de la protección internacional para hacer frente a los nuevos desafíos que el cambio climático nos presenta.

Por último, cabe señalar -tal como fue desarrollado en el texto- que el obstáculo más complejo que observamos para la aplicación de protección complementaria por motivos climáticos, está en la idea de que el retorno a un lugar en donde la persona se ve privada de una serie de derechos como consecuencia de un desastre natural, pueda configurarse como un trato cruel, inhumano o degradante. Esto, porque a la luz del derecho internacional y la jurisprudencia existente los tratos crueles, inhumanos y degradantes se han entendido, generalmente, como efecto del actuar del hombre. Sin perjuicio de ello, creemos que es un campo en el que puede profundizarse y que invita a explorar la posibilidad de ampliar su alcance en un estudio posterior.

\section{Referencias}

ACNUR (1998). Conclusiones sobre la Protección Internacional de los Refugiados aprobadas por el Comité Ejecutivo, núm. 85 (XLIX). Conclusión General sobre la Protección Internacional. Disponible en https://bit.ly/307fTV6.

-. (1992). Manual de procedimientos y criterios para determinar la condición de refugiado en virtud de la Convención de 1951 y el Protocolo de 1967, sobre el Estatuto de los Refugiados. Disponible en https://bit.ly/3mXzWnG.

-. (2005a). Introducción a la Protección Internacional Protección de las personas de la competencia del ACNUR: Módulo autoformativo 1. Disponible en https://www. refworld.org.es/docid/4714bdo82.html. 
-. (2005b). Providing International Protection Including Through Complementary Forms of Protection (EC/55/SC/CRP.16). Disponible en http://bit.ly/3aXP2ar.

ACNUR (2007). Opinión Consultiva sobre la aplicación extraterritorial de las obligaciones de no devolución en virtud de la Convención sobre el Estatuto de los Refugiados de 1951 y su Protocolo de 1967, párr. 6. Disponible en https://bit.ly/38PACGH.

Bou Franch, Valentín y Mireya Castillo Daudí (2010). Curso de derecho internacio-

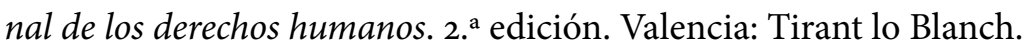

Conferencia Internacional sobre Refugiados Centroamericanos (1994). Evaluación de la puesta en práctica de las disposiciones del Documento: «Principios y criterios para la protección y asistencia a los refugiados, repatriados $y$ desplazados Centroamericanos en América Latina». Disponible en https://www. refworld.org/docid/3ae6b31c88.html.

Cooper, Jessica B. (1998). Environmental Refugees: Meeting the Requirements of the Refugee Definition. Nueva York: University Environmental Law Journal.

Corte IDH (2013). Caso Familia Pacheco Tineo vs. Estado Plurinacional de Bolivia. Excepciones Preliminares, Fondo, Reparaciones y Costas, sentencia del 25 de noviembre del 2013, párr. 145, disponible en <http://bit.ly/2MlOGAc.

CORTE IDH (2018). La institución del asilo y su reconocimiento como derecho humano en el Sistema Interamericano de Protección (interpretación y alcance de los artículos 5, 22.7 y 22.8, en relación con el artículo 1.1 de la Convención Americana sobre Derechos Humanos), Opinión Consultiva OC-25/18 de 30 de mayo de 2018, disponible en https://www.corteidh.or.cr/docs/opiniones/seriea_25_esp.pdf.

EDWARDS, Alice (2006). «Refugee Status Determination in Africa». African Journal of International and Comparative Law, 14: 204-233.

García, Gonzalo y Pablo Contreras (2014). «Diccionario constitucional chileno». Cuadernos del Tribunal Constitucional, 55: 50-52.

Goodwin-Hill, Guy S. (2014). The International Law of Refugee Protection. Editado por Elena Fiddian-Qasmiyeh y otros. Oxford. Oxford University Press. Disponible en https://bit.ly/2XocBqY.

Hathaway, James C. (1991). The Law of Refugee Status. Toronto: Butterworths.

Henríquez, Miriam (2019). Acción de amparo. Santiago: DER Ediciones.

KäLIN, Walter (2010). Climate Change and Displacement: Multidisciplinary Perspectives. Chapter 5. Conceptualising Climate. Induced Displacement. Editado por Jane McAdam. Oxford and Portland, Oregon: Hart Publishing.

KNox, John H. (2019). «Issue of human rights obligations relating to the enjoyment of a safe, clean, healthy and sustainable environment: Report of the Special Rapportear». Oficina del Alto Comisionado de Naciones Unidas para los Derechos Humanos (ACNUDH). Disponible en https://bit.ly/34P1oPZ. 
Kozoll, Christopher M. (2004). «Poisoning the Well: Persecution, the Environment, and Refugee Status». Colorado Journal of International Environmental Law and Policy, 15 (2): 271-307.

McAdam, Jane (2012). Climate Change, Forced Migration, and International Law. Oxford: Oxford University Press.

McAdam, Jane (2007). Complementary Protection in International Refugee Law. Oxford: Oxford University Press.

McBeth, Adam, Justine Nolan y Simon Rice (2018). The International Law of Human Rights. Oxford: Oxford University Press.

Nogueira Alcalá, Humberto (2002). «La libertad personal y las dos caras de Jano en el ordenamiento jurídico chileno». Revista de Derecho (Universidad Austral de Chile), 13: 161-186. Disponible en http://revistas.uach.cl/index.php/revider/ article/view/2787/2370.

Reyes Canales, Gabriela y Beatriz Romero (2018). La protección complementaria y el Estatuto de Refugiados, alcance y factibilidad de su aplicación en Chile. Santiago: Facultad de Derecho de la Universidad de Chile.

Schwartz, Michelle. 1993. "International Legal Protection for Victims of Environmental Abuse». Yale Journal of International Law, 18: 355-380.

Sferrazza, Pietro y Francisco Bustos (2019). La constitucionalización del derecho a no ser sometido a torturas. Coordinado por Gonzalo Aguilar. Nuevos Derechos para una Nueva Constitución. Valencia: Tirant lo Blanch.

Simeon, James C. (2013). The UNHCR and the Supervision of International Refugee Law. Cambridge: Cambridge University Press.

SмIтH, Rhona (2016). International Human Rights Law. Oxford: Oxford University Press.

STOREY, Hugo (2014). «What constitutes persecution? Towards a working definition». International Journey of Refugee Law. Oxford Academic, 26 (2): 272-285. Disponible en https://doi.org/10.1093/ijrl/eeuo17.

VILLÁn DuRÁN, Carlos (1985). «La convención contra la tortura y su contribución a la definición del derecho a la integridad física y moral en el derecho internacional». Revista Española de Derecho Internacional, 37 (2): 377-402.

VIllá́n Durán, Carlos (1998). «La obligatoriedad jurídica de la Declaración Universal», en Xavier Pons Rafols (coord.), La Declaración Universal de los Derechos Humanos (pp. 73-89). Barcelona: Icaria Antrazyn.

\section{Sobre los autores}

Francisco Bustos Bustos es abogado. Licenciado en Ciencias Jurídicas y Sociales por la Universidad de Chile, académico del Departamento de Enseñanza Clínica del Derecho, en la Facultad de Derecho de la Universidad de Chile, Chile. Colaborador 
de Observatorio de Justicia Transicional (Universidad Diego Portales, Chile). Se desempeña en el Estudio Jurídico Caucoto Abogados. Su correo electrónico es fbustos@ derecho.uchile.cl. (D) https://orcid.org/0000-0003-1013-7206.

Beatriz Romero Cruzat es abogada. Licenciada en Ciencias Jurídicas y Sociales por la Universidad de Chile, exconsultora del Alto Comisionado de Naciones Unidas para los Refugiados (ACNUR) y exoficial de protección en el Departamento de Extranjería y Migraciones (DEM). Actualmente, se desempeña como consultora para el Programa Asilo de Calidad en México. Su correo electrónico es bearomeroc@ ug.uchile.cl. (D) https://orcid.org/oooo-0002-1178-0167. 
La Revista de Derecho Ambiental, del Centro de Derecho Ambiental de la Facultad de Derecho de la Universidad de Chile, es un espacio de exposición y análisis en el plano académico del derecho ambiental. Su contenido se presenta a través de doctrina, jurisprudencia y recensiones, y aborda diversas materias relacionadas con la gestión, institucionalidad y herramientas de protección ambiental y desarrollo sustentable. Se presentan artículos de diferentes autores y autoras en los que se analizan y abordan casos y temas jurídico-ambientales de creciente interés y actualidad.

\author{
DIRECTORA \\ Valentina Durán Medina \\ EDITORES \\ Jorge Ossandón Rosales \\ y Antonio Pulgar Martínez \\ SITIO WEB \\ revistaderechoambiental.uchile.cl \\ CORREO ELECTRÓNICO \\ revistada@derecho.uchile.cl \\ LICENCIA DE ESTE ARTÍ́CULO \\ Creative Commons Atribución Compartir Igual 4.o Internacional
}

La edición de textos, el diseño editorial

y la conversión a formatos electrónicos de este artículo

estuvieron a cargo de Tipográfica

(www.tipografica.io) 